\title{
Gut Microbiome Diversity and Specific Composition During Immunotherapy in Japanese Responders with Non-Small Cell Lung Cancer
}

Fumihiro Shoji ( $\nabla$ fshoji@surg2.med.kyushu-u.c.jp )

National Hospital Organization, Kyushu Medical Center

Masafumi Yamaguchi

National Hospital Organization, Kyushu Cancer Center

Masaki Okamoto

National Hospital Organization, Kyushu Medical Center

Shinkichi Takamori

National Hospital Organization, Kyushu Cancer Center

Yuka Kozuma

National Hospital Organization, Kyushu Medical Center

Gouji Toyokawa

National Hospital Organization, Kyushu Medical Center

Koji Yamazaki

National Hospital Organization, Kyushu Medical Center

Tatsuro Okamoto

National Hospital Organization, Kyushu Cancer Center

Mitsuhiro Takenoyama

National Hospital Organization, Kyushu Cancer Center

Mototsugu Shimokawa

Yamaguchi University

Sadanori Takeo

National Hospital Organization, Kyushu Medical Center

Yoshihiko Maehara

Kyushu Central Hospital

\section{Research Article}

Keywords: Oral microbiome, gut microbiome, diversity, specific composition, non-small cell lung cancer (NSCLC), immune checkpoint inhibitors response

Posted Date: June 28th, 2021 
DOI: https://doi.org/10.21203/rs.3.rs-633455/v1

License: (c) (1) This work is licensed under a Creative Commons Attribution 4.0 International License. Read Full License 


\section{Abstract}

Cancer immunotherapy including immune checkpoint inhibitors (ICI) has revolutionized non-small cell lung cancer (NSCLC) therapy. Recently, the microbiome status before initiation of ICl therapy has been emphasized as a predictive biomarker in patients undergoing ICI therapy. However, the microbiome diversity and composition during ICl therapy is unknown. This multicenter, prospective observational study analyzed both saliva and feces from 28 patients with NSCLC. We performed $16 \mathrm{~S}$ ribosomal RNA gene sequencing, then analyzed associations of oral and gut microbiome diversity or composition with $\mathrm{ICI}$ response. Seventeen patients (responders) had a partial ICI response, and the remaining 11 patients (non-responders) had stable or progressive disease. At the genus level, the alpha diversity of the gut microbiome was significantly greater in ICI responders than in non-responders (Chao 1, $p=0.0174$; PD whole tree, $p=0.0219$; observed species, $p=0.0238$; Shannon, $p=0.0362$ ), while the beta diversity of the gut microbiome was significantly lower in ICI responders (principal coordinates analysis, $p=0.035$ ). Compositional differences in the gut microbiome were observed between the two groups; in particular, Blautia was enriched in ICl responders, whereas RF32 unclassified was enriched in ICl non-responders. There were no significant differences between groups in the oral microbiome. This study revealed a strong association between gut microbiome diversity and ICI response in Japanese NSCLC patients. Moreover, specific gut microbiome compositions may influence the $\mathrm{ICI}$ response. These findings might be useful in identifying biomarkers to predict $\mathrm{ICl}$ response, as well as in developing biotic therapies to enhance the $\mathrm{ICl}$ response.

\section{Background}

Immunotherapy with immune checkpoint inhibitors (ICI) is widely used to treat various malignancies, including non-small cell lung cancer (NSCLC); it has revolutionized therapeutic approaches to cancer. Programmed death-ligand-1 (PD-L1) is an immune checkpoint protein expressed on tumor cells and tumor-infiltrating immune cells, which can mediate anticancer immunosuppression [1]. Anti-PD-1 antibodies (e.g., nivolumab and pembrolizumab) and anti-PD-L1 antibodies (e.g., atezolizumab and durvalumab) enable T-cell activation and immune system recognition.

Although tumorous PD-L1 expression is a potential biomarker of the ICI therapeutic response, there is no widely accepted optimal biomarker to predict the efficacy of $\mathrm{ICl}$, because $\mathrm{ICI}$ response and survival outcomes show heterogeneity in NSCLC patients receiving ICl therapy, regardless of PD-L1 expression level.

We recently reported that the pretreatment host immune-nutritional condition was a prognostic marker for NSCLC patients receiving ICl therapy [2]. Host immunity is clearly associated with the ICI response. The internal microbiome is regarded as a controlling factor in host immunity. In particular, the gut microbiome can modulate the host immune response (e.g., anti-tumor immunity) and optimize both innate and adaptive immune responses [3]. Recently, preclinical studies have shown that the gut microbiome composition and its modification in murine models could influence the efficacy of $\mathrm{ICI}[4,5]$. Therefore, the 
microbiome has been emphasized as a predictive biomarker of ICI therapy, mainly in studies from the USA or Europe. Additionally, the gut microbiome diversity or abundance of specific gut microbiome components has been correlated with the efficacy of anti-PD-1 antibody in melanoma patients [6]. Moreover, fecal microbiome transplantation (FMT) in murine models might restore the ICI response [7, 8]. In a recent study, FMT from $\mathrm{ICI}$ responders to $\mathrm{ICI}$ non-responders produced ICl efficacy in melanoma patients [9].

Furthermore, the oral microbiome has been associated with several diseases (e.g., inflammatory bowel disease and allergic diseases) through its influence on the gut microbiome [10-12]. A recent study revealed that variation in the oral microbiome was associated with a risk of lung cancer [13]. However, samples were collected prior to ICl therapy in most previous studies, and thus minimal information has been available regarding the microbiome status during ICI therapy. Accordingly, FMT or biotics therapy approaches are needed to investigate changes in the microbiome during $\mathrm{ICl}$ therapy. Notably, there are definite differences in microbiome composition among ethnicities [14]; to the best of our knowledge, few reports have been published regarding Japanese NSCLC patients. Here, we performed a prospective study to clarify the microbiome diversity and composition in Japanese NSCLC patients by analyzing samples collected during $\mathrm{ICl}$ therapy.

\section{Results}

\section{Patient characteristics}

The results were determined in follow-up examinations over a mean duration of 598 days (range, 811225 days) after initial ICl therapy. Patient characteristics are shown in Table 1. The study group included seven women and 21 men, with a mean age at surgery of 71 years (range, 56-88 years). Fifteen patients (53.6\%) had ECOG performance status (PS) 0 and 13 (46.4\%) had ECOG-PS 1. Seven patients (25.0\%) had never smoked, and the remaining 21 patients were current or FORMER smokers. The histological types were adenocarcinoma in 16 patients (57.1\%) and squamous cell carcinoma in 12 patients (42.9\%). Of the 28 included patients, one (3.6\%) had stage IIA, seven (25.0\%) had stage III (two with IIIA, three with IIIB, and two with IIIC), 11 (39.3\%) had stage IV (seven with IVA and four with IVB), and nine (32.1\%) had postoperative recurrence. Seven patients $(25.0 \%)$ had mutant epithelial growth factor receptor (EGFR) and 21 patients (75.0\%) had wild-type EGFR or no data regarding EGFR status. ICI was first-line therapy in 11 patients $(39.3 \%)$, second-line therapy in 10 patients $(35.7 \%)$, third-line therapy in five patients $(17.8 \%)$, and fourth-line or later therapy in two patients $(7.2 \%)$. Twenty-four patients $(85.7 \%)$ received ICI monotherapy (nivolumab: $3 \mathrm{mg} / \mathrm{kg}$ or $240 \mathrm{mg} /$ body intravenously at 2-week intervals; pembrolizumab: $200 \mathrm{mg} /$ body intravenously at 3-week intervals; atezolizumab: $1200 \mathrm{mg} /$ body intravenously at 3-week intervals; or durvalumab: $10 \mathrm{mg} / \mathrm{kg}$ intravenously at 2-week intervals), while the remaining four patients received ICl therapy combined with platinum-doublet chemotherapy (pembrolizumab: $200 \mathrm{mg} /$ body plus carboplatin, area under the curve [AUC] for concentration-time: $5 \mathrm{mg} / \mathrm{mL} /$ minute; plus pemetrexed $500 \mathrm{mg} / \mathrm{m}^{2}$ or nabpaclitaxel $200 \mathrm{mg} / \mathrm{m}^{2}$ intravenously at 3-week intervals). Ten patients (35.7\%) had more than $50 \%$ 
tumorous PD-L1 expression, 10 patients (35.7\%) had 1-49\% PD-L1 expression, and eight patients (28.6\%) had no PD-L1 expression or no data regarding PD-L1 status.

Table 1

Patient characteristics

\begin{tabular}{|lll|}
\hline Items & & No. $(\%)$ or Median (Range) \\
\hline Gender & Male/female & $21(75.0) / 7(25.0)$ \\
\hline Age & Year & $71(56-88)$ \\
\hline ECOG-PS & $\mathbf{0} 1$ & $15(53.6) / 13(46.4)$ \\
\hline Smoking status & Current/former/non & $12(42.9) / 9(32.1) / 7(25.0)$ \\
\hline Stage & Advanced/postoperative recurrence & $19(67.9) / 9(32.1)$ \\
\hline Pathology & $\begin{array}{l}\text { Adenocarcinoma/Squamous cell } \\
\text { carcinoma }\end{array}$ & $16(57.1) / 12(42.9)$ \\
\hline EGFR status & Mutant type/wild type or not evaluated & $7 / 21$ \\
\hline PD-L1 expression & $>$ 50/1-49/<1/unknown & $10(35.7) / 10(35.7) / 3(10.8) / 5(17.8)$ \\
\hline ICl & Monotherapy & $24(11 / 3 / 7 / 3)(85.7)$ \\
\hline Line & Combination therapy & $4(14.3)$ \\
\hline Cycles & $\mathbf{1 / 2 / 3 / 4 / > 5}$ & $10(35.7) / 10(35.7) / 6(21.4) / 1(3.6) / 1$ \\
\hline Response & CR/PR/SD/PD & $20(3-81)$ \\
\hline
\end{tabular}

Abbreviations: CR: complete response; ECOG: European Clinical Oncology Group; EGFR: epidermal growth factor receptor; ICl: immune-check point inhibitors; PD: progressive disease; PD-L1: programmed deathligand 1; PS: performance status; PR: partial response; SD: stable disease.

\section{ICl response in NSCLC patients}

No patient experienced a complete response (CR) (0\%), 17 patients had a partial response (PR) $(60.7 \%)$, two patients had stable disease (SD) (7.2\%), and nine patients (32.1\%) had progressive disease (PD). Therefore, 17 patients (PR) were regarded as ICl responders; the remaining 11 patients (SD or PD) were regarded as $\mathrm{ICl}$ non-responders (Supplementary Fig. 1).

\section{Relative abundances in oral and gut microbiomes}

We analyzed the relative abundances of oral bacteria at the phylum and genus levels. At the phylum level, Firmicutes, Bacteroides, Proteobacteria, Actinobacteria, Fusobacteria, and TM 7 were the main taxa; these 
taxa comprised more than $99 \%$ in all groups. At the genus level, 169 species were detected in the ICl responder saliva microbiome, while 152 species were detected in the $\mathrm{ICI}$ non-responder saliva microbiome (Fig. 2A, B). Among the taxa with $<1 \%$ relative abundance, 157 taxa (92.9\%) were identified in the $\mathrm{ICl}$ responder saliva microbiome, while 138 taxa $(90.8 \%)$ were identified in the ICI non-responder saliva microbiome. Taxa in both groups mainly included Streptococcus, Veillonella, Prevotella, Haemophilus, and Neisseria (Fig. 2C, D). Regarding the gut microbiome, at the phylum level, Firmicutes, Bacteroides, Actinobacteria, Proteobacteria, Fusobacteria, and Verrucomicrobia were the main taxa (comprising more than 99\%) in ICl responders. Firmicutes, Bacteroides, Actinobacteria, Proteobacteria and Fusobacteria were the main taxa (comprising more than 99\%) in ICI non-responders. At the genus level, 180 species were detected in the $\mathrm{ICI}$ responder gut microbiome, while 136 species were detected in the $\mathrm{ICl}$ nonresponder gut microbiome (Fig. 2E, F). Among the taxa with $<1 \%$ relative abundance, 167 taxa $(92.8 \%)$ were identified in the $\mathrm{ICI}$ responder gut microbiome, while 122 taxa $(89.7 \%)$ were identified in the $\mathrm{ICI}$ nonresponder gut microbiome. Taxa in $\mathrm{ICl}$ responders mainly included Bacteroides, Ruminococcaceae, Lachnospiraceae, Streptococcus, and Blautia; taxa in ICI non-responders mainly included Bacteroides, Ruminococcaceae, Lachnospiraceae, Prevotella, and Bifidobacterium (Fig. 2G, H).

\section{Diversity metrics in oral and gut microbiomes}

We used alpha and beta diversity indices to evaluate the intersample and intrasample relationships in the oral and gut microbiomes. Figure 3 and Fig. 4 show the alpha diversity metrics of oral and gut microbiomes at the genus level. In the oral microbiome, there were no significant differences between the two groups (Chao 1, $p=0.632$; Observed species, $p=0.523$; PD_whole_tree, $p=0.471$; and Shannon, $p=$ 0.474 ) (Fig. 3A-D). In the gut microbiome, all alpha diversity metrics were significantly higher in ICI responders than in $\mathrm{ICl}$ non-responders (Chao $1, \mathrm{p}=0.017$; Observed species, $\mathrm{p}=0.024$; PD_whole_tree, $\mathrm{p}=$ 0.022; and Shannon, $p=0.036$ ) (Fig. $3 \mathrm{E}-\mathrm{H})$. PCoA assessment of beta diversity was conducted based on weighted UniFrac distance (Fig. 4). In both oral and gut microbiomes, similar patterns were evident in the two groups. The greatest variations in the oral and gut microbiomes of the two groups were $4.39 \%$ (PC1) and $4.32 \%$ (PC2), and $4.51 \%$ (PC1) and 4.22\% (PC2), respectively. PERMANOVA based on unweighted UniFrac distance confirmed significant differences between the two groups in the gut microbiome alone (Fig. 4A, oral: $p=0.904$ and Fig. 4C, gut: $p=0.035$ ). Additionally, boxplot analyses showed significant differences in the gut microbiome alone (Fig. 4B, oral: $p=0.760$ and Fig. 4D, gut: $p=0.005$ ).

\section{LEfSe results}

We used LEfSe to perform high-dimensional genus comparisons regarding oral and gut microbiomes between $\mathrm{ICl}$ responders and non-responders. Figure 5 shows that the $\mathrm{ICI}$ responder gut microbiome was significantly enriched for Blautia, compared with the ICI non-responder gut microbiome. In contrast, the ICI non-responder gut microbiome was significantly enriched for RF32 unclassified, compared with the ICI responder gut microbiome. There were no significant differences in the oral microbiome between the two groups. 


\section{Discussion}

Similar studies regarding $\mathrm{ICl}$ therapy have focused on the microbiome in patients who have not yet received ICl therapy. Those studies revealed that the microbiome diversity and composition before ICI therapy was a predictive biomarker for ICI response. Although variations in gut microbiome composition were observed in the previous studies, there has been minimal information regarding gut microbiome status during $\mathrm{ICl}$ therapy. This information is important for efforts to enhance $\mathrm{ICl}$ therapy through biotics therapy (e.g., pre-, pro-, and synbiotics) and/or FMT. This analysis of oral and gut microbiome profiles in Japanese NSCLC patients during ICI therapy produced several novel findings.

The gut microbiome might have an important role in the ICI response in NSCLC patients, although the oral microbiome conveyed information distinct from the gut microbiome. Greater numbers of both oral and gut microbiome species were observed in $\mathrm{ICI}$ responders than in $\mathrm{ICI}$ non-responders. Additionally, those microbiomes mainly consisted of minor species $(<1 \%)$ at the genus level. Moreover, the 4 th and 5 th majority of gut microbiome species in ICl responder were differed from that in ICI non-responder, while 1st to 5 th majority of oral microbiome were same between two groups.

An important tool for objective evaluation of the above data involves analysis of microbiome diversity: the numbers or abundances of microorganisms colonizing the gut. Greater alpha diversity indicates larger numbers of species in the gut, which implies a distinct gut microbiome composition. Several studies have reported that the $\mathrm{ICl}$ response was influenced by the alpha diversity of the gut microbiome before ICI therapy $[8,27]$. In the present study, high alpha diversity was observed in the ICI responder gut microbiome. Thus, our results indicated that ICI responders had more abundant gut microbes, compared with ICl non-responders, during ICI therapy.

Our study also revealed a significant association between beta diversity and ICI response. Higher beta diversity indicates a significant difference in gut microbiome composition between two samples. In this study, the intersample distance was significantly shorter in $\mathrm{ICl}$ responders than in ICI non-responders. Therefore, the ICI responder gut microbiome had significant similarity, compared with the ICI nonresponder gut microbiome, which implies a simple approach to control the gut microbiome by adding biotics therapy to $\mathrm{ICl}$ therapy.

Additionally, we identified specific gut microbiome species in Japanese NSCLC patients receiving ICI therapy by using LEfSe. The results indicated that Blautia was enriched in ICI responders, whereas RF32 unclassified was enriched in ICI non-responders. Several studies have shown significant associations between various gut microbiome components (e.g., Bifidobacterium longum [6], Collinsella aerofaciens [6], Enterococcus faecium [6], Akkermansia muciniphila [7], Ruminococcaceae [8], Faecalibacterium genii [29] and Firmicutes [29]) and clinical response to ICI therapy. These results differed among individual studies, including the present study, presumably due to factors such as patient disease (e.g., melanoma, renal cell carcinoma, and NSCLC) and ICI regimen. Indeed, Frankel et al [28] indicated that the gut microbiome composition depended on the $\mathrm{ICl}$ regimen. Moreover, these differences might be caused by ethnicities. Nishijima et al [14] compared the gut microbiome between the Japanese population and 
individuals from 11 other nations. Notably, the Japanese gut microbiome was considerably different from the microbiomes of other populations; it was characterized by the highest abundances of Blautia, Bifidobacterium, Collinsella, Streptococcus, and an unclassified Clostridiales genus, compared with the microbiomes from the remaining 11 countries.

In addition to its status as a species characteristic of the gut microbiome in Japanese individuals, Blautia coccoides is regarded as an effective probiotic species. B. coccoides is an anaerobe and Gram-positive species found in human fecal samples. Reduced numbers of $B$. coccoides are associated with several benign diseases (e.g., hepatic cirrhosis and encephalopathy, irritable bowel syndrome, acute diarrhea, idiopathic inflammatory bowel disease, intestinal inflammation, and diabetes mellitus) and some malignancies (e.g., colorectal and breast cancers) [30, 31]. Furthermore, some reports have revealed that increased numbers of $B$. coccoides might be beneficial for human health. $B$. coccoides is increased among individuals with diets high in resistant starch and arabinoxylan [32]; moreover, it can reduce NF-KB activity in human colon cancer cells [33]. Additionally, Blautia obeum is a gut microbiome component involved in the transformation of carcinogenic heterocyclic amines, and reduced abundance of this species may increase heterocyclic amine-induced colorectal cancer risk [34]. Myles et al [35] reported that high omega-3 intake altered colonic inflammation and increased Blautia abundance in a murine model. Therefore, Blautia may be a key gut microbiome component involved in the ICI response in Japanese NSCLC patients. Conversely, the relative abundance of RF32 unclassified has been positively correlated with colonic damage and inflammation [36], which are presumably negative influences on immunity in NSCLC patients.

The findings in this study indicate that controlling both gut microbiome diversity and the abundances of specific gut microbiome species during $\mathrm{ICl}$ therapy might lead to $\mathrm{ICl}$ response enhancement in Japanese NSCLC patients. Future therapies targeting the gut microbiome by means of pre-, pro-, or synbiotics to enhance the $\mathrm{ICl}$ response might be considered from our findings.

This study had the following limitations. First, only a single sample collection was performed. Thus, it was unclear how the microbiome diversity and composition might have changed before and after $\mathrm{ICl}$ therapy. Second, the sample size was small, which may have interfered with meaningful conclusions. Third, this study allowed various $\mathrm{ICl}$ regimens with or without combination chemotherapy. In the future, we plan to perform a large, multicenter, prospective observational study to evaluate the association between $\mathrm{ICl}$ response and changes in gut microbiome by collecting samples at multiple points (before and during ICI therapy) for NSCLC patients receiving a specific ICl regimen.

In conclusion, this study revealed a strong association between gut microbiome diversity and ICI response in Japanese NSCLC patients. Moreover, specific gut microbiome compositions may influence the $\mathrm{ICl}$ response. These findings might be useful in identifying biomarkers to predict $\mathrm{ICl}$ response, as well as in developing biotic therapies to enhance the $\mathrm{ICl}$ response.

\section{Materials And Methods}




\section{Study design and participants}

This prospective observational study was conducted at multiple centers: Department of Thoracic Surgery and Department of Respiratory Medicine, Clinical Research Institute, National Hospital Organization (NHO)Kyushu Medical Center and Department of Thoracic Oncology, NHO Kyushu Cancer Center. This study was approved by the ethics committee of Kyushu Medical Center (approval number, 19C031) and the ethics committee of Kyushu Cancer Center (approval number, 2019-44). All research activities comply with all relevant ethical regulations and were performed in accordance with relevant guidelines and regulation in Kyushu Medical Center and Kyushu Cancer Center. Written informed consent to use samples and clinico-pathological data for research purposes was obtained from all participants. Eligibility criteria were as follows: pathologically or cytologically confirmed diagnosis of locally advanced/unresectable or postoperative recurrent NSCLC; receipt of ICI monotherapy including nivolumab (Opdivo, Bristol-Myers Squibb), pembrolizumab (Keytruda, Merck), atezolizumab (Tecentriq, Genentech), and durvalumab (Imfinzi, Astra Zeneca) or platinum-based therapy combined with these ICl agents. Patients were also enrolled if they had discontinued these therapies but had not received any additional therapies.

From July 2019 to December 2020, 34 NSCLC patients were eligible and enrolled. Of those 34 patients, 28 had both saliva and feces samples available for this study (Supplementary Fig. 1). All enrolled patients had at least one measurable target lesion based on the Response Evaluation Criteria in Solid Tumors (RECIST), version 1.1 [15]. Clinical/pathological stage was based on the Tumor Node Metastasis (TNM) classification established by the International Union Against Cancer [16]. For TNM staging, all patients underwent computed tomography (CT) of the thorax and upper abdomen, as well as bone scintigrams, brain CT scans, magnetic resonance imaging (MRI), or fluorodeoxyglucose-positron emission tomography (FDG-PET). Postoperative local or distant recurrence was defined as described previously [17]. ICI therapy was continued until radiographic progression. PD-L1 protein expression was evaluated using antibody clone 22C3 (Dako, Agilent Technologies, Santa Clara, CA, USA).

\section{Sample Collection}

Salivary and fecal samples were collected in sterile containers and immediately placed at $4^{\circ} \mathrm{C}$, then frozen at $-80^{\circ} \mathrm{C}$. Individual periods of sample collection are shown in Fig. 1. The mean numbers of days between the initiation of ICI therapy and the day of sample collection were 307 (29-945) days in ICI responders and $117(23-491)$ days in $\mathrm{ICI}$ non-responders.

\section{DNA extraction, gene amplification, sequencing, and data analysis procedures}

Preliminary treatment of fecal samples was conducted in accordance with a previously described method [18]; DNA was then extracted using an automated DNA isolation system (Gene Prep Star PI-480, Kurabo, Japan). DNA was extracted from saliva using the Mora-Extract kit (Kyokuto Pharmaceutical, Japan). The V3-V4 regions of bacterial 16S rRNA genes were amplified using the Pro341F/Pro805R primers [18] and 
dual-index method [19] under hemi-nested PCR conditions [20]. Barcoded amplicons were paired-end sequenced on a 2×284-bp cycle using the MiSeq system with MiSeq Reagent Kit chemistry, version 3 (600 Cycle). Paired-end sequencing reads were merged using the fastq-join program with default settings [21]. Only reads with quality value $(\mathrm{QV})$ scores of $\geq 33$ were extracted with split_libraries_fastq.py command in QIIME, version 1.8.0 [22]. Chimeric sequences were removed using USEARCH61 [23] with the identify_chimeric_seqs.py command in QIIME [22]. Operational taxonomic units (OTUs) were aligned using the pick_open_reference_otus.py command in QIIME [22]. OTUs with 97\% similarity were identified with the Greengenes database, version 13.8 [24]. Alpha diversity indices (e.g., observed species, Chao-1, Shannon, and PD_whole_tree) and beta diversity indices (e.g., principal coordinates analysis [PCoA]) were analyzed using the alpha_rarefaction.py and beta_diversity.py commands in QIIME, respectively [23]. The Chao-1 index was used to determine community richness and the Shannon index was used to determine community diversity. The PD_whole_tree index was used to compute Faith's phylogenetic diversity. PCoA was used to show differences between the two groups. Unweighted UniFrac metrics were used for beta diversity [25]. Linear discriminant analysis effect size (LEfSe) measurements were used to quantify differential taxonomic and functional pathway abundances between responders and non-responders [26].

\section{Statistical analysis}

Categorical variables were analyzed using Fisher's exact test. Continuous variables were compared using the chi-squared test. Statistical analyses were performed using JMP software, version 14.0 (SAS Institute, Inc., Cary, NC, USA). The adonis function in the vegan package of $\mathrm{R}$ software, version 3.6.1, was used to conduct permutational multivariate analysis of variance (PERMANOVA) with respect to microbiome composition. $P$-values $<0.05$ were considered statistically significant.

\section{Declarations}

\section{Acknowledgements}

We thank Ryan Chastain-Gross, Ph.D., from Edanz (https://jp.edanz.com/ac) for editing a draft of this manuscript.

\section{Author contributions}

Study concept and design (F.S., M.Y., S.T. and Y.M.); acquisition of data (F.S., M.O., S.T., Y.K., G.T., K.Y. and T.O.); analysis and interpretation of data (F.S., M.Y. and M.T.); statistical analysis (M.S.); drafting of the manuscript (F.S., S.T. and Y.M.).

\section{Funding Supports}

This study was supported by JSPS KAKENHI Grant Number JP20K09188 and the Japanese Foundation for Multidisciplinary Treatment of Cancer (JFMC).

\section{Competing interests}


All authors have declared no conflicts of interest.

\section{References}

1. Ribas A, et al. Cancer immunotherapy using checkpoint blockade. Science 359: 1350-1355 (2018).

2. Shoji $F$, et al. Pretreatment prognostic nutritional index as a novel biomarker in non-small cell lung cancer patients treated with immune checkpoint inhibitors. Lung Cancer 136: 45-51 (2019).

3. Hooper LV, et al. Interactions between the microbiota and the immune system. Science. 336: 1268-73 (2012).

4. Vétizou M, et al. Anticancer immunotherapy by CTLA-4 blockade relies on the gut microbiota. Science 350: 1079-84 (2015).

5. Sivan A, et al. Commensal Bifidobacterium promotes antitumor immunity and facilitates anti-PD-L1 efficacy. Science 350: 1084-9 (2015).

6. Matson $\mathrm{V}$, et al. The commensal microbiome is associated with anti-PD-1 efficacy in metastatic melanoma patients. Science 359: 104-108 (2018).

7. Routy B, et al. Gut microbiome influences efficacy of PD-1-based immunotherapy against epithelial tumors. Science 359: 91-97 (2018).

8. Gopalakrishnan V, et al. Gut microbiome modulates response to anti-PD-1 immunotherapy in melanoma patients. Science 359: 97-103 (2018).

9. Davar D, et al. Fecal microbiota transplant overcomes resistance to anti-PD-1 therapy in melanoma patients. Science 371: 595-602 (2021).

10. Atarashi $\mathrm{K}$, et al. Ectopic colonization of oral bacteria in the intestine drives $\mathrm{T}_{\mathrm{H}^{1}}$ cell induction and inflammation. Science 358: 359-365 (2017).

11. El-Solh AA, et al. Microbiology of severe aspiration pneumonia in institutionalized elderly. Am J Respir Crit Care Med 167: 1650-4 (2003).

12. Yamasaki K, et al. Significance of anaerobes and oral bacteria in community-acquired pneumonia. PLoS One 8: e63103 (2013).

13. Hosgood HD, et al. Variation in oral microbiome is associated with future risk of lung cancer among never-smokers. Thorax 76: 256-263 (2021).

14. Nishijima $S$, et al. The gut microbiome of healthy Japanese and its microbial and functional uniqueness. DNA Res 23: 125-33 (2016).

15. Eisenhauer EA, et al. New response evaluation criteria in solid tumours: revised RECIST guideline (version 1.1). Eur J Cancer 45: 228-47 (2009).

16. Goldstraw P, et al. The IASLC Lung Cancer Staging Project: Proposals for Revision of the TNM Stage Groupings in the Forthcoming (Eighth) Edition of the TNM Classification for Lung Cancer. J Thorac Oncol 11: 39-51 (2016). 
17. Varlotto JM, et al. Factors associated with local and distant recurrence and survival in patients with resected nonsmall cell lung cancer. Cancer 115: 1059-69 (2009).

18. Takahashi S, et al. Development of a prokaryotic universal primer for simultaneous analysis of Bacteria and Archaea using next-generation sequencing. PLoS One 9:e105592 (2014).

19. Hisada T, et al. Inter-and intra-individual variations in seasonal and daily stabilities of the human gut microbiota in Japanese. Arch Microbiol 197:919-934 (2015).

20. Hell $\mathrm{K}$, et al. The dynamic bacterial communities of a melting High Arctic glacier snowpack. ISME J 7: 1814-1826 (2013)

21. Aronesty E. Comparison of sequencing utility programs. Open Bioinforma J 7: 1-8 (2013).

22. Caporaso JG, et al. QIIME allows analysis of high-throughput community sequencing data. Nat Methods 7: 335-336 (2010).

23. Edgar RC, Haas BJ, Clemente JC, Quince C, Knight R. UCHIME improves sensitivity and speed of chimera detection. Bioinformatics 27: 2194-2200 (2011).

24. DeSantis TZ, et al. Greengenes, a chimera-checked 16S rRNA gene database and workbench compatible with ARB. Appl Environ Microbiol 72: 5069-5072 (2006).

25. Lozupone $C A$, et al. Quantitative and qualitative beta diversity measures lead to different insights into factors that structure microbial communities. Appl Environ Microbiol 73: 1576-85 (2007).

26. Segata N, et al. Metagenomic biomarker discovery and explanation. Genome Biol 12: R60 (2011)

27. Jin Y, et al. The Diversity of Gut Microbiome is Associated With Favorable Responses to AntiProgrammed Death 1 Immunotherapy in Chinese Patients With NSCLC. J Thorac Oncol 14: 13781389 (2019).

28. Frankel $A E$, et al. Metagenomic Shotgun Sequencing and Unbiased Metabolomic Profiling Identify Specific Human Gut Microbiota and Metabolites Associated with Immune Checkpoint Therapy Efficacy in Melanoma Patients. Neoplasia 19: 848-855 (2017).

29. Chaput N, et al. Baseline gut microbiota predicts clinical response and colitis in metastatic melanoma patients treated with ipilimumab. Ann Oncol 28: 1368-1379 (2017).

30. Chen W, et al. Human intestinal lumen and mucosa-associated microbiota in patients with colorectal cancer. PLoS One 7: e39743 (2012).

31. Mabrok HB, et al. Lignan transformation by gut bacteria lowers tumor burden in a gnotobiotic rat model of breast cancer. Carcinogenesis 33: 203-8 (2012).

32. Nielsen TS, et al. Diets high in resistant starch and arabinoxylan modulate digestion processes and SCFA pool size in the large intestine and faecal microbial composition in pigs. Br J Nutr 112: 1837-49 (2014).

33. Lakhdari O, et al. Identification of NF-KB modulation capabilities within human intestinal commensal bacteria. J Biomed Biotechnol 2011: 282356 (2011).

34. Katsidzira L, et al. Differences in Fecal Gut Microbiota, Short-Chain Fatty Acids and Bile Acids Link Colorectal Cancer Risk to Dietary Changes Associated with Urbanization Among Zimbabweans. Nutr 
Cancer 71: 1313-1324 (2019).

35. Myles IA, et al. Effects of parental omega-3 fatty acid intake on offspring microbiome and immunity. PLoS One 9: e87181 (2014).

36. Castro-Mejía J, et al. Treatment with a Monoclonal Anti-IL-12p40 Antibody Induces Substantial Gut Microbiota Changes in an Experimental Colitis Model. Gastroenterol Res Pract 2016: 4953120 (2016).

\section{Figures}

Figure 1

\begin{tabular}{|c|c|c|c|c|c|c|c|c|}
\hline Group & RECIST & Gender & Stage & His & PD-L1 & ICI & Line & Regimen \\
\hline$\overline{\mathrm{R}}$ & PR & $\mathrm{M}$ & $\mathrm{Rec}$ & $\mathrm{Ad}$ & 0 & $\mathrm{M}$ & 2 & Nivolumab \\
\hline $\mathrm{R}$ & PR & $\mathrm{F}$ & Adv & Ad & $1-49$ & $\mathrm{M}$ & 2 & Pembrolizumab \\
\hline $\mathrm{R}$ & PR & M & Adv & $\mathrm{Sq}$ & $>50$ & $\mathrm{M}$ & 3 & Pembrolizumab \\
\hline $\mathrm{R}$ & PR & $\mathrm{F}$ & Adv & Ad & $\mathrm{NE}$ & $\mathrm{M}$ & 5 & Nivolumab \\
\hline $\mathrm{R}$ & PR & $\mathrm{M}$ & $\operatorname{Rec}$ & $\mathrm{Sq}$ & $>50$ & $\mathrm{M}$ & 1 & Atezolizumab \\
\hline $\mathrm{R}$ & $\mathrm{PR}$ & $\mathrm{M}$ & Adv & $\mathrm{Sq}$ & $>50$ & $\mathrm{M}$ & 1 & Pembrolizumab \\
\hline $\mathrm{R}$ & PR & M & Rec & Ad & $>50$ & $\mathrm{M}$ & 3 & Nivolumab \\
\hline $\mathrm{R}$ & PR & $\mathrm{M}$ & Adv & Ad & $1-49$ & $\mathrm{M}$ & 2 & Pembrolizumab \\
\hline $\mathrm{R}$ & PR & $\mathrm{M}$ & Adv & $\mathrm{Sq}$ & $>50$ & $\mathrm{M}$ & 1 & Pembrolizumab \\
\hline$R$ & PR & $\mathrm{M}$ & Adv & $\mathrm{Sq}$ & $1-49$ & $\mathrm{M}$ & 1 & Pembrolizumab \\
\hline $\mathrm{R}$ & PR & M & Adv & $\mathrm{Sq}$ & $\mathrm{NE}$ & $\mathrm{C}$ & 1 & CBDCA/nab-PTX/Pembro \\
\hline $\mathrm{R}$ & PR & $\mathrm{F}$ & Adv & Ad & $>50$ & $\mathrm{M}$ & 1 & Pembrolizumab \\
\hline $\mathrm{R}$ & PR & $\mathrm{M}$ & Adv & $\mathrm{Sq}$ & $1-49$ & $\mathrm{M}$ & 2 & Pembrolizumab \\
\hline $\mathrm{R}$ & PR & M & Adv & $\mathrm{Sq}$ & $1-49$ & $\mathrm{C}$ & 1 & CBDCA/nab-PTX/Pembro \\
\hline $\mathrm{R}$ & PR & $\mathrm{M}$ & Adv & Ad & $>50$ & $\mathrm{M}$ & 2 & Durvalumab \\
\hline $\mathrm{R}$ & PR & $\mathrm{M}$ & Adv & Ad & $>50$ & $\mathrm{M}$ & 3 & Atezolizumab \\
\hline $\mathrm{R}$ & PR & $\mathrm{F}$ & Adv & Ad & $\mathrm{NE}$ & $\mathrm{M}$ & 2 & Durvalumab \\
\hline NR & $\mathrm{SD}$ & $\mathrm{M}$ & Adv & $\mathrm{Sq}$ & $1-49$ & $\mathrm{M}$ & 2 & Pembrolizumab \\
\hline NR & PD & $\mathrm{M}$ & Rec & $\mathrm{Sq}$ & $>50$ & $\bar{c}$ & 1 & CBDCA/nab-PTX/Pembro \\
\hline NR & PD & $\mathrm{M}$ & Adv & Ad & $1-49$ & $\mathrm{C}$ & 1 & CBDCA/Pem/Pembro \\
\hline NR & PD & M & Rec & $\mathrm{Sq}$ & $1-49$ & $\mathrm{M}$ & 2 & Pembrolizumab \\
\hline $\mathrm{NR}$ & SD & $F$ & Adv & Ad & $1-49$ & $\mathrm{M}$ & 2 & Durvalumab \\
\hline NR & PD & $\mathrm{F}$ & Rec & Ad & $>50$ & $\mathrm{M}$ & 1 & Pembrolizumab \\
\hline NR & PD & $\mathrm{M}$ & Rec & Ad & $\mathrm{NE}$ & $\mathrm{M}$ & 4 & Atezolizumab \\
\hline NR & PD & $\mathrm{M}$ & Rec & Ad & 0 & $\mathrm{M}$ & 3 & Atezolizumab \\
\hline NR & PD & $\mathrm{M}$ & Rec & Ad & $\mathrm{NE}$ & $\mathrm{M}$ & 3 & Atezolizumab \\
\hline NR & PD & $\mathrm{F}$ & Adv & Ad & $\mathrm{NE}$ & $\mathrm{M}$ & 2 & Nivolumab \\
\hline $\mathrm{NR}$ & PD & M & Adv & $\mathrm{Sq}$ & $1-49$ & $\mathrm{M}$ & 2 & Atezolizumab \\
\hline
\end{tabular}

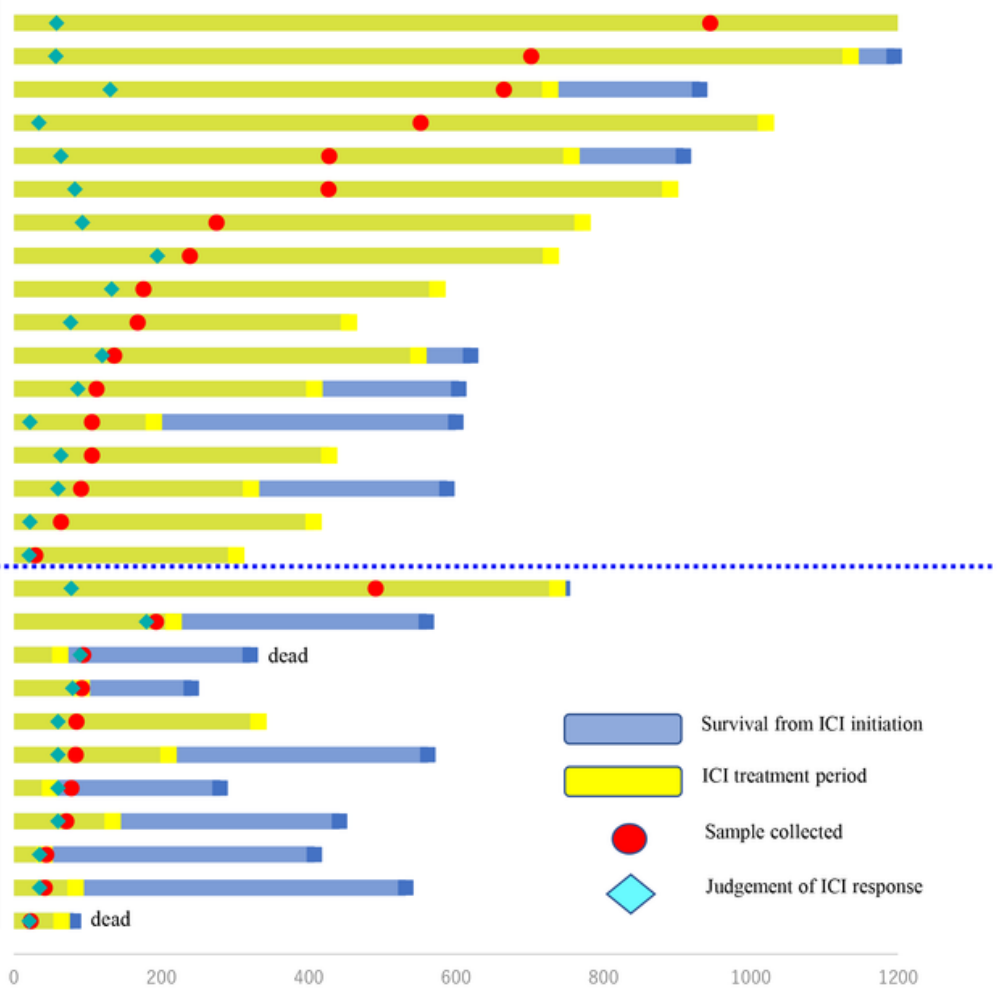

\section{Figure 1}

Swimmer plot of survival after initiation of ICI therapy. Each bar represents one patient. The left column shows clinicopathological characteristics. 
Figure 2

A.

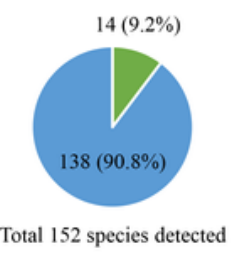

C.

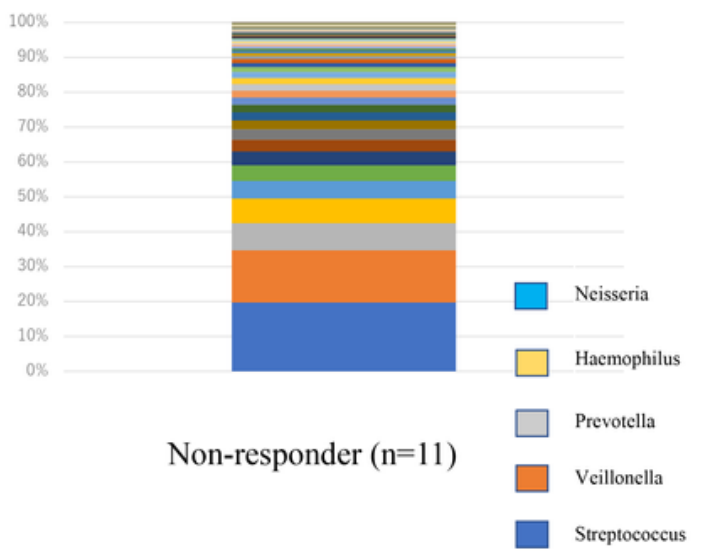

E.

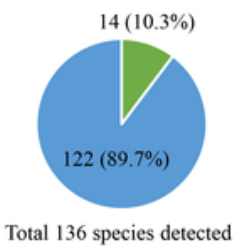

G.

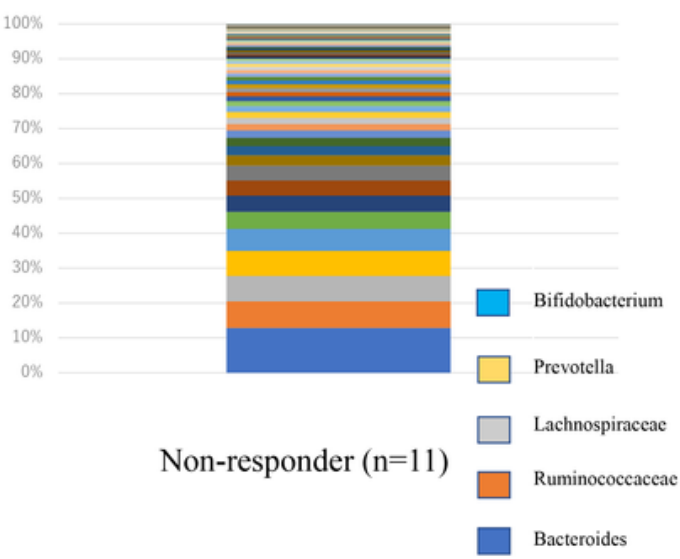

B.

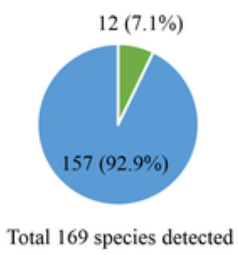

D.
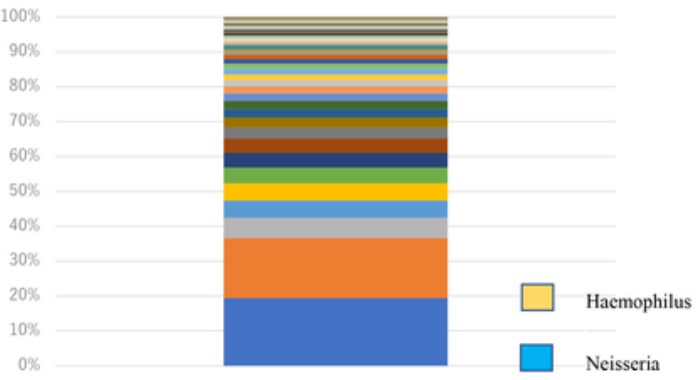

Responder $(\mathrm{n}=17)$

Prevotella

$\square$ Veillonella

Streptococcus
F.

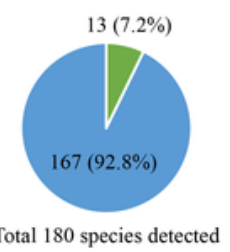

H.

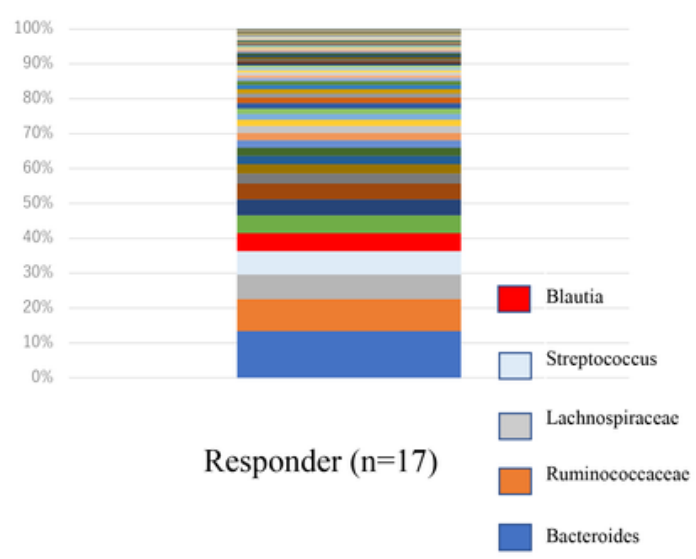

\section{Figure 2}

Comparisons of taxa relative abundances between $\mathrm{ICI}$ responders and non-responders at genus level. (A and $B$ ) Distributions of oral microbiome taxa relative abundances $>1 \%$ and $<1 \%$ in ICl non-responders (A) and responders (B). (C and D) Comparisons of oral microbiome taxa relative abundances in ICI nonresponders (C) and responders (D). (E and F) Distributions of gut microbiome taxa relative abundances 
$>1 \%$ and $<1 \%$ in $\mathrm{ICl}$ non-responders $(\mathrm{E})$ and responders $(\mathrm{F})$. ( $\mathrm{G}$ and $\mathrm{H})$ Comparisons of gut microbiome taxa relative abundances in $\mathrm{ICl}$ non-responders $(\mathrm{G})$ and responders $(\mathrm{H})$.

Figure 3
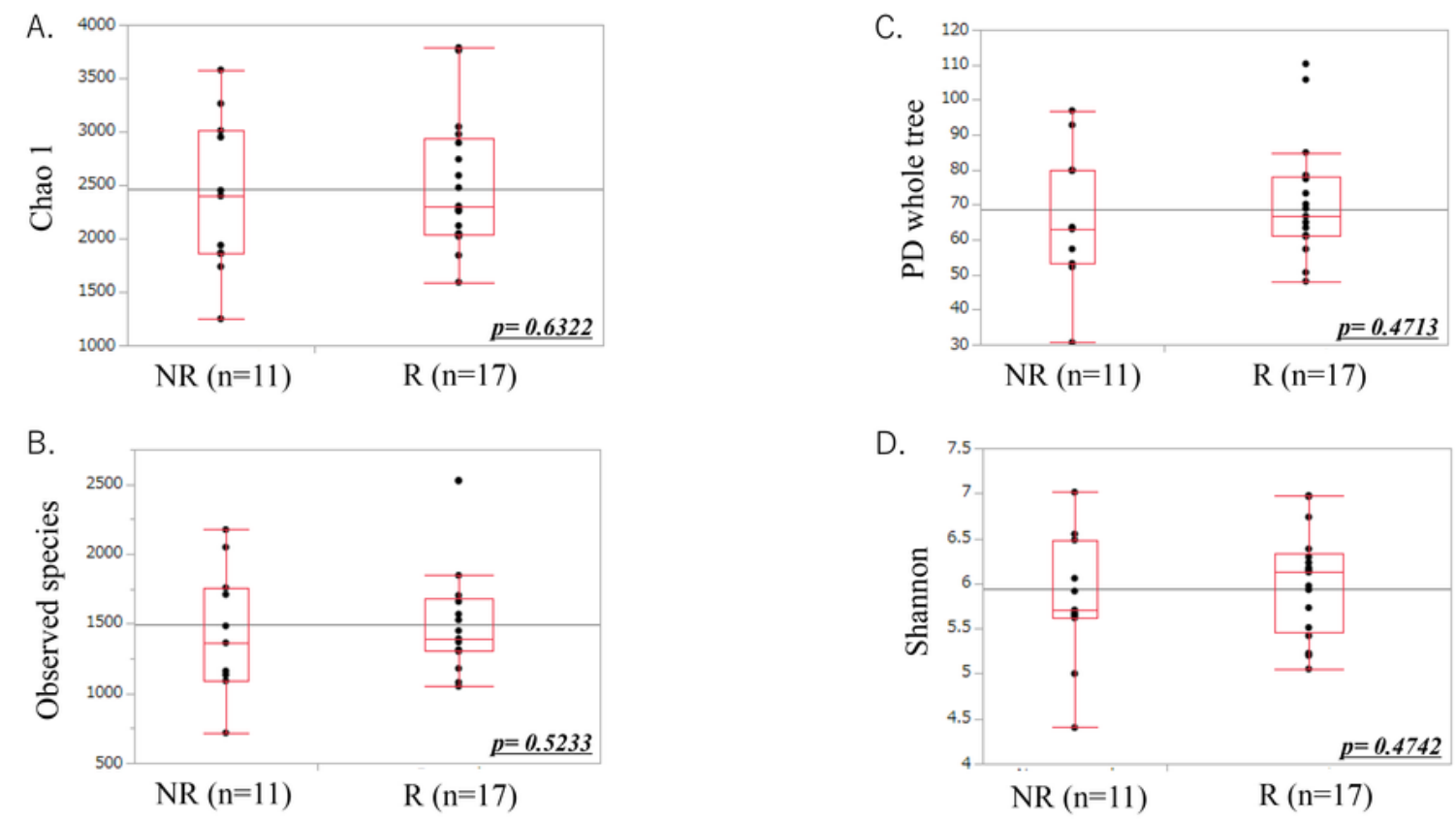

E.

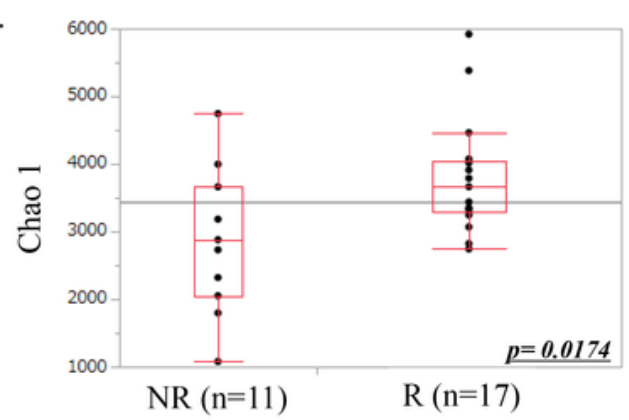

F.

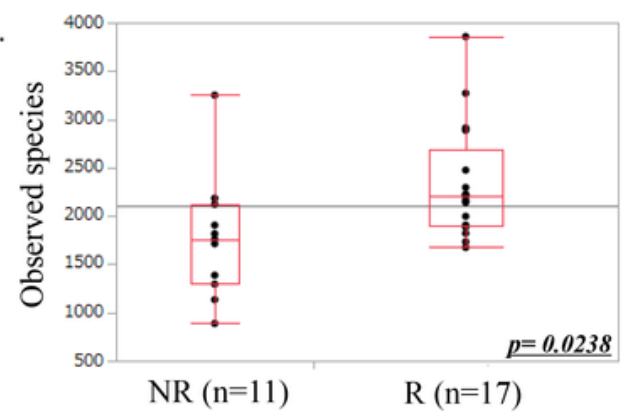

G.

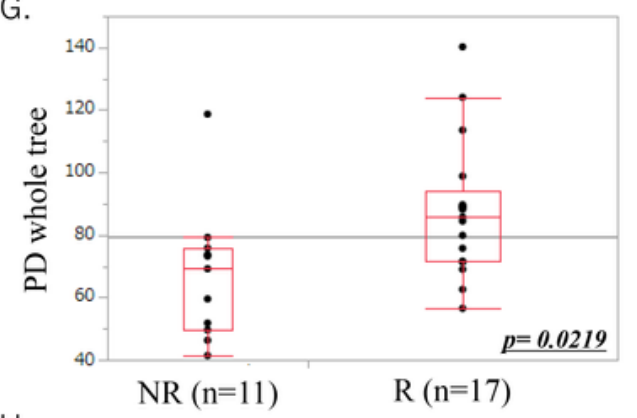

н.

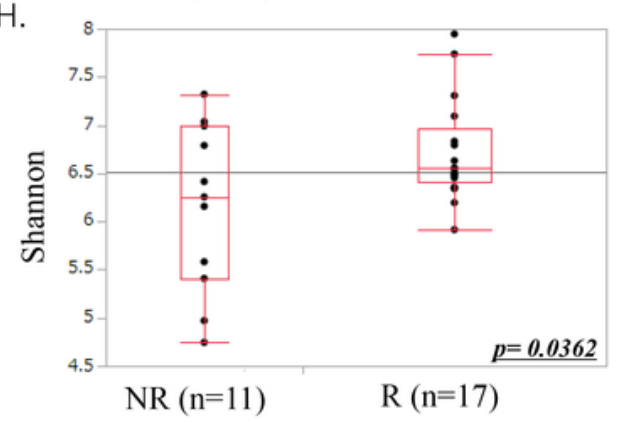

\section{Figure 3}

Comparisons of oral (A-D) and gut (E-H) microbiome diversities between ICI non-responders and responders. (A and $E)$ Chao1 index, (B and F) observed species, (C and G) PD_whole_tree, and (D and H) Shannon index. 
Figure 4

A.

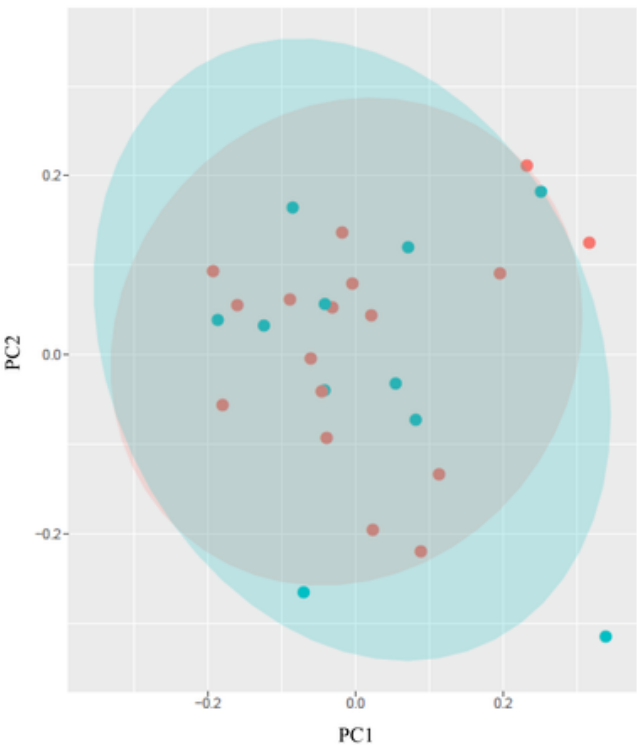

C.

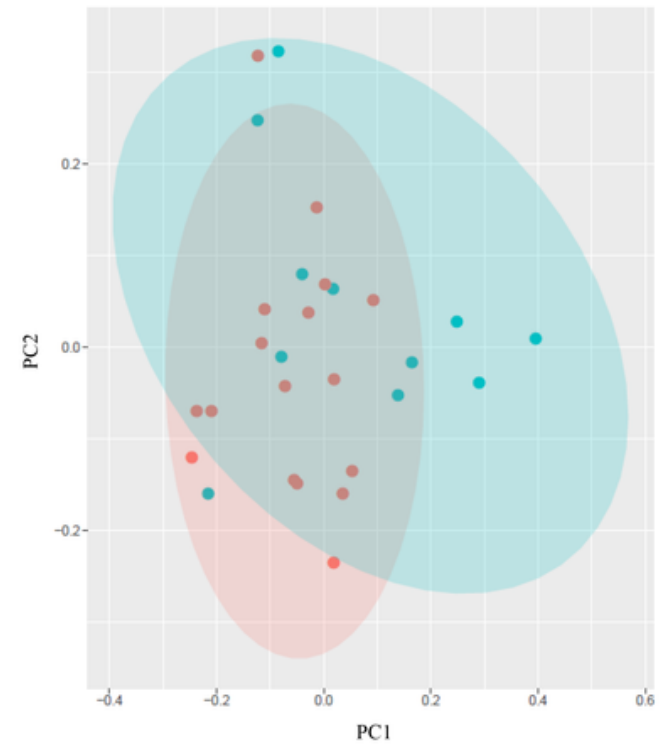

B.

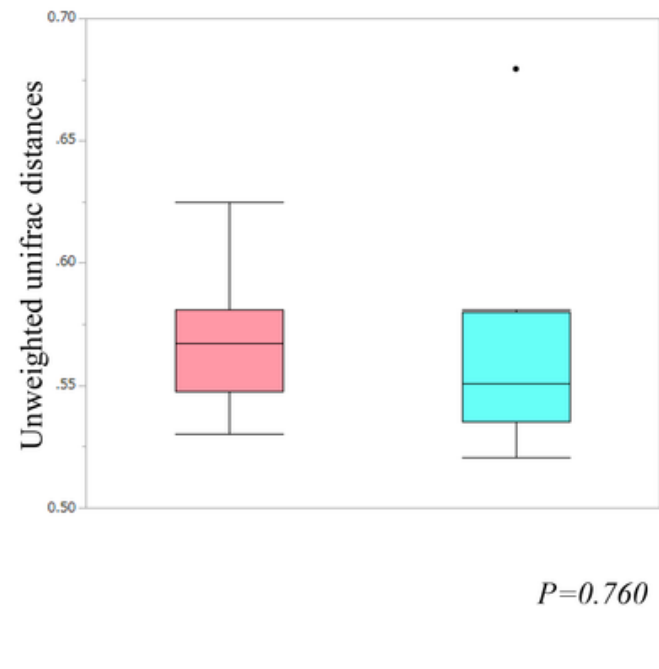

D.

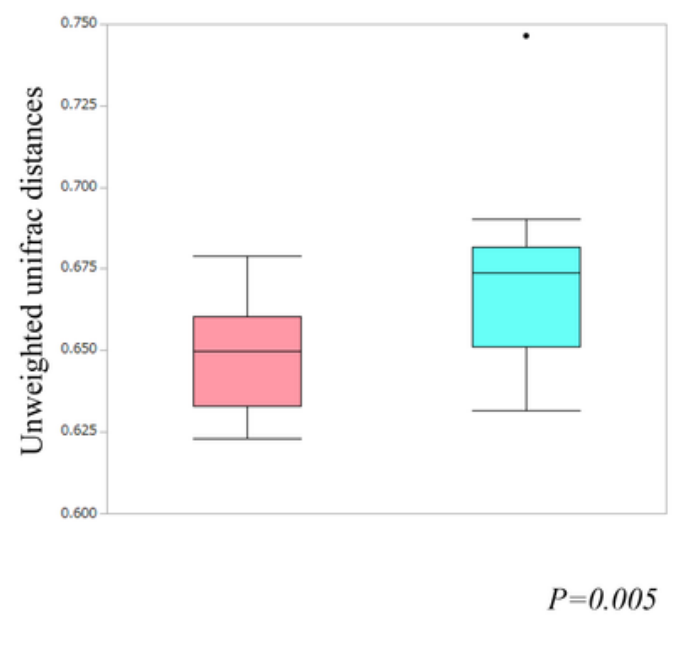

\section{Figure 4}

Principal coordinates analysis ( $A$ and $C$ ) and boxplots ( $B$ and $D$ ) of microbiome data based on unweighted UniFrac distances between ICI non-responders and responders at the genus level. 
Figure 5

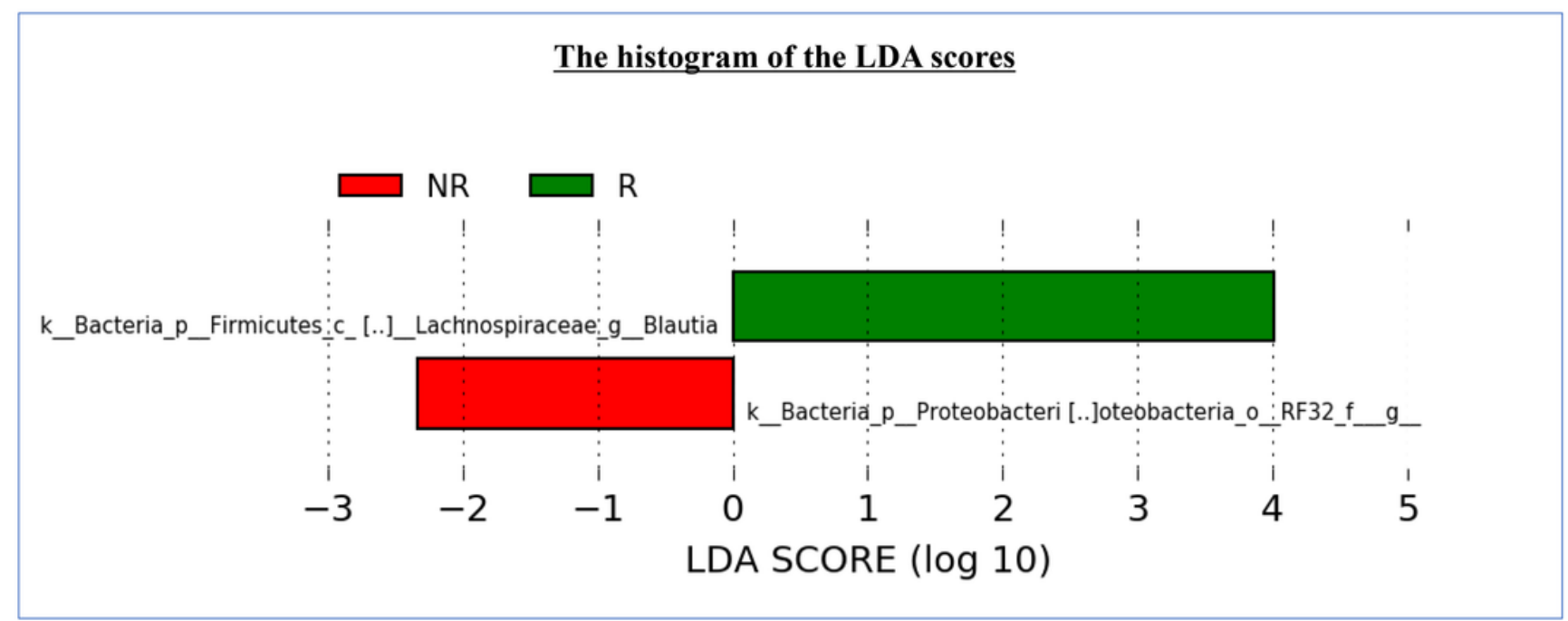

\section{Figure 5}

Results of LEfSe analysis of differentially abundant taxa in gut microbiomes between ICI responders and non-responders.

\section{Supplementary Files}

This is a list of supplementary files associated with this preprint. Click to download.

- SuppleFigure1.pptx 